inflammation through regulation of cytokine, chemokine and metalloprotease expression. Thus, the study of the non-canonical pathway of Wnt5a in the aggressive phenotype of FLS and the inflammatory response may provide new therapeutic targets for the RA.

Objectives: To analyse the involvement of Wnt5a in the proliferation, migration, invasion and inflammatory responses of FLS from RA patients.

Methods: FLS were obtained from eight RA patients. Expression of Wnt5a was suppressed by siRNA transfection (Dharmacon). Cellular proliferation was determined using the CellTiter-Glo Luminescent Cell Viability Assay (Promega). Migration was analysed by the wound-healing assay using lbidi inserts. The occupation area was determined by Image J. Invasion was tested by quantifying Giemsa stained cells on the bottom side of Matrigel coated trans-membrane (Millipore). The expression of inflammatory mediators and metalloproteases was analysed by real-time PCR after stimulation with recombinant Wnt5a protein (rWnt5a), TNF and combined treatment.

Results: We analysed the effect of the absence of Wnt5a and rWnt5a on the proliferation, migration and invasion of RA FLS. FLS lacking Wnt5a showed a significant migration decrease, $27.9 \%$ in basal condition and $16.9 \%$ after TNF stimulation, when compared with FLS transfected with siRNA control. Accordingly, migration of RA FLS treated with rWnt5a was increased by $48.5 \%$ when compared with cells treated with the vehicle. No change in migration was observed in TNF-stimulated FLS. Invasive capacity was reduced, reaching $71.4 \%$ of that observed in FLS transfected with siRNA control in basal conditions. In addition, invasion in FLS treated with $\mathrm{rWnt5}$ a was $35.7 \%$ higher than in controls. We also analysed the effect of rWnt5a on the inflammatory response of RA FLS. We found a significantly increase of IL6, IL8, CCL2, CXCL5, MMP9 and MMP13 basal expression and the TNF-induced expression of IL6, IL8, CXCL5 and MMP3. Nevertheless, the lack of Wnt5a or the addition of rWnt5a did not modify the spontaneous or the TNF-induced proliferation.

Conclusions: These results indicate that Wnt5a contributes to the aggressive phenotype of FLS by potentiating their migration and invasion, and by stimulating the inflammatory response.

Acknowledgements: Supported by grant of the ISCIII/PI14/01660/RETICS Program, RD16/0012/0014/cofinanced FEDER

Disclosure of Interest: None declared

DOI: 10.1136/annrheumdis-2018-eular.4538

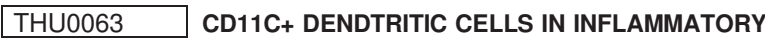 ARTHRITIS}

A. Puchner ${ }^{1}$, V. Saferding ${ }^{1}$, M. Bonelli ${ }^{1}$, H. Leiss ${ }^{1}$, R. Pfeifle ${ }^{2}$, G. Kroenke ${ }^{2}$ J. Smolen ${ }^{1}$, K. Redlich ${ }^{1}$, S. Blüml ${ }^{1}{ }^{1}$ Medical University of Vienna, Vienna, Austria; ${ }^{2}$ University Hospital Erlangen, Erlangen, Germany

Background: Dendritic cells (DCs) are important antigen presenting cells (APCs) and therefore they play an important role in bridging the innate and the adaptive immune response. DCs can be divided in different subsets with specific functions. As powerful APCs, DCs are thought to play an important role in the induction of autoimmune diseases such as rheumatoid arthritis. However, the active role of DCs in joint inflammation is not known yet.

Objectives: We analysed histological sections of $\mathrm{K} / \mathrm{BxN}$ serum transfer arthritis as well as hTNFtg arthritis for the presence of CD11c+cells by immunohistochemistry. We also performed synovial biopsies and analysed the cellular composition of the inflammatory infiltrate with respect to DCs. We used CD11c-diphteria toxin receptor (DTR) transgenic mice, which express the human diphtheria- toxin receptor under the CD11c promoter, allowing for specific depletion of CD11c +cells by administration of diphtheria toxin (DT). K/BxN serum transfer arthritis was induced, and mice were given either DT or PBS or in wt and BARF3 deficient mice. In addition CD11c DTR mice were crossed into hTNFtg animals and also received either DT or PBS. The severity of arthritis was determined clinically and histologically.

Methods: We analysed histological sections of $\mathrm{K} / \mathrm{BxN}$ serum transfer arthritis as well as hTNFtg arthritis for the presence of CD11c+cells by immunohistochemistry. We also performed synovial biopsies and analysed the cellular composition of the inflammatory infiltrate with respect to DCs. We used CD11c-diphteria toxin receptor (DTR) transgenic mice, which express the human diphtheria- toxin receptor under the CD11c promoter, allowing for specific depletion of CD11c +cells by administration of diphtheria toxin (DT). K/BxN serum transfer arthritis was induced, and mice were given either DT or PBS or in wt and BARF3 deficient mice. In addition CD11c DTR mice were crossed into hTNFtg animals and also received either DT or PBS. The severity of arthritis was determined clinically and histologically.

Results: We show that $\mathrm{Cd} 11 \mathrm{c}+$ cells are present in significant numbers in the synovia of K/BxN and TNF driven arthritis. Both CD8 +CD11c+and CD11b + CD11C+, can be found in synovial tissue. Upon depletion of CD11c+cells clinical signs of $\mathrm{K} / \mathrm{BxN}$ serum transfer arthritis were significantly reduced. Histological analysis found reduced synovial inflammation after the depletion of CD11c+cells in $\mathrm{K} / \mathrm{BxN}$ arthritis. In addition, local bone destruction and the number of osteoclasts was also significantly reduced. Analysis of $\mathrm{K} / \mathrm{BxN}$ arthritis in wt mice and BATF3-/- mice, which lack a subset of DCs, namely CD8 +CD11+DCs, revealed no difference in arthritis severity between the two groups. In addition to $\mathrm{K} / \mathrm{BxN}$ arthritis, we found that also in TNF-driven arthritis depletion of CD11c+cells led to a striking reduction of synovial inflammation and a complete depletion of osteoclasts.

Conclusions: These data show that in addition to initiating an adaptive immune response, CD11c+dendritic cells, are also involved in innate effector mechanisms of inflammatory arthritis. Especially CD11b+CD11c+and monocyte derived inflammatory seem to play a role in inflammatory arthritis, suggesting that they could be an important therapeutic target for patients suffering from inflammatory arthritis.

Disclosure of Interest: None declared

DOI: 10.1136/annrheumdis-2018-eular.7320

\section{THU0064 ANTIBODIES AGAINST CARBAMYLATED PROTEINS FROM PATIENTS WITH RHEUMATOID ARTHRITISACTIVATE ENDOTHELIAL CELLS}

A. Pecani, T. Colasanti, F.R. Spinelli, C. Barbati, F. Conti, G. Valesini, C. Alessandri. Department of Internal Medicine and Medical Specialties, Rheumatology Unit, Sapienza University of Rome, Roma, Italy

Background: Inflammation contributes to the excess of cardiovascular morbidity in rheumatoid arthritis (RA), by promoting endothelial activation; this brings toward the production of adhesion molecules and the activation of signalling mediators Antibodies against carbamylated proteins (anti-CarP) detected in RA patients correlate with subclinical atherosclerosis.

Objectives: Aims of the present study were: 1) to determine the effect of antiCarP antibodies purified from the sera of RA patients, on the production of VCAM1 and ICAM1 as well as the activation of IRAK1 and NF-kB by human endothelial cell line EAhy926. 2) To evaluate endothelial cell apoptosis induced by antiCarP

Methods: An indirect ELISA was used to detect the presence of anti-CarP in the sera of RA patients. To purify anti-CarP, carbamylated-FCS used as an antigen was spotted onto a nitrocellulose filter and incubated with patient's sera that recorded the highest titer. Antibodies were eluted with glycine $100 \mathrm{mM}, \mathrm{pH} 2.5$ and neutralised with Tris- $\mathrm{HCl} 1 \mathrm{M}, \mathrm{pH} 8$. Antibodies concentration was measured by using a colorimetric Bradford assay. The immortalised hybridoma cell line EAhy926 was cultured in Dulbecco's Modified Medium containing 10\% fetal bovine serum, $1 \mathrm{mM}$ l-glutamine, $100 \mathrm{U} / \mathrm{ml}$ penicillin and $10 \mathrm{ml} \mathrm{HAT}$. After cell stimulation with purified anti-CarP at different time points (30 min-48 h) and different concentrations $(5-20-50 \mu \mathrm{g} / \mathrm{ml})$, supernatants were gathered to investigate the production of VCAM-1, ICAM-1 using commercial ELISA kits while activation of IRAK1 and NF-kB was detected by Western Blot analysis using cell lysates. Apoptosis was measured using FITC-conjugated annexin $\mathrm{V}(\mathrm{AV})$ and a propidium iodide (PI) apoptosis detection kit at different times (30 min-48 h).

Results: After EAhy926 stimulation with anti-CarP we observed: 1) induction of VCAM-1 but not ICAM production in cell supernatants; 2 ) activation of IRAK1 and NFkB transcription factor in cell lysates and 3 ) induction of endotelial cell apoptosis.
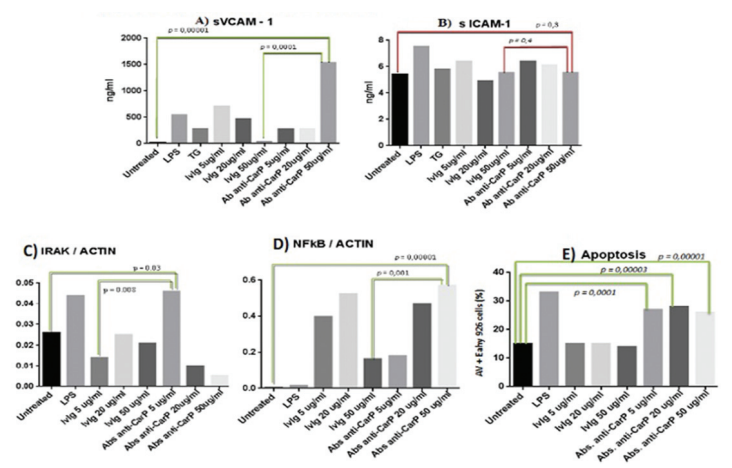

Abstract THU0064 - Figure 1. Expression of addhesion molecules (A, B), activation of IRAK (C), NFkB (D) signalling and apoptosis (E) in EAhy 926.

Conclusions: These data suggest a potential involvement of anti-CarP antibodies in endothelial cell activation allowing to further speculate on its possible effect in excess cardiovascular risk in RA patients.

REFERENCES:

[1] Pecani A, et al. Arthritis Res Ther 2016; 
[2] Spinelli FR, Pecani A, et al., BMC Musculosceletal Disord 2017;

[3] Gonzales-Gay MA, et al. Clin and Exp Rheumatol 2006;

[4] Jing Shi, et al. Autoimmun Rev 2014.

Acknowledgements: . EULAR Scientific Bursaries, Programme of 2017

Disclosure of Interest: None declared

DOI: 10.1136/annrheumdis-2018-eular.6217

\section{THU0065 RESULTS OF TREATMENT OF ARTHROSCOPIC AND TRADITIONAL SYNOVECTOMY OF THE KNEE JOINT IN RHEUMATOID ARTHRITIS}

A. Kasimov, I. Khujanazarov. Traumatology, Tashkent Medical Academy, Tashkent, Uzbekistan

Background: Rheumatoid arthritis is a systemic inflammatory autoimmune disease of connective tissue with a predominant joint injury that occurs at any age, more often in women. It is characterised by a chronic course with periods of exacerbations and remissions

Objectives: To evaluate the results of arthroscopic and traditional synovectomy of the knee joint in patients with rheumatoid arthritis

Methods: The study involved 48 patients, 29 of them were women and 19 men, aged from 25 to 65 years old, who had a chronic synovitis of the knee joints. It was implemented at the department of traumatology, orthopaedics and GPH with neurosurgery of Tashkent Medical Academy and 1-Republic Clinical Hospital. In all patients, on the background of basic therapy and intraarticular injections of SCS, a persistent recurrent synovitis was formed within a few months. Sixteen patients underwent sinocapsullectomy (SCE) with the traditional method. 32 patients underwent arthroscopic synovectomy (ASE), for which the standard basic arthroscopic technique Dyonics (Smith and Nephew) was used. The functional condition of the knee joint before and after the operation was assessed using the KOOS scale (Knee and Osteoarthritis Outcome Score)

Results: The study showed that movements in the operated joint were allowed 3-5 hours after the operation, from the first day, isometric exercises were prescribed, active movements in the knee joint before the onset of pain. Results of treatment in terms of up to 3 months were tracked in all 48 patients. The degree of intraarticular changes determined by arthroscopy was evaluated according to the Outerbridge classification. In patients after SCE, pain in the joint, limiting the amplitude of motion, crunching during movement were noted. After ASE, there was a significant improvement in the function and condition of the knee joint: patients could walk for longer distances without additional support, did not experience discomfort while walking the stairs, complaints of pain, and no swelling. Repeated ASE with tunnelling was required in 2 patients with grade III chondromalacia

Conclusions: Thus, the SCE of the knee is indicated if the conservative treatment of recurrent synovitis in RA is unsuccessful for a long time. The ASE should be performed in the early stages of the RA, which drastically reduces the activity of the pathological process, makes it possible to maintain the functional capacity of the joint, and the timely adequate administration of the basic therapy allows the maximum achieved long-term functional result to be maintained for the longest time and reduces the risk of relapse and the need for repeated operations

\section{REFERENCES:}

[1] Steultjens EEMJ, et al. Occupational therapy for rheumatoid arthritis. Cochrane Database of Systematic Reviews 2009.

[2] Sweeney SE, et al. Clinical features of rheumatoid arthritis. In GS Firestein, et al., eds., Kelley's Textbook of Rheumatology 2013(9th ed.);2:1109-1136. Philadelphia: Saunders.

Disclosure of Interest: None declared

DOI: 10.1136/annrheumdis-2018-eular.6999

\section{THU0066 CIRCULATING MIRNAS AS POTENTIAL BIOMARKERS OF DISEASE AND CARDIOVASCULAR RISK IN RHEUMATOID ARTHRITIS PATIENTS}

C. López-Pedrera ${ }^{1}$, N. Barbarroja ${ }^{1}$, P. Ruiz-Limón ${ }^{1}$, S. Remuzgo-Martínez ${ }^{2}$, I. Arias de la Rosa ${ }^{1}$, M.C. Ábalos-Aguilera ${ }^{1}$, Y. Jimenez-Gomez ${ }^{1}$, R. Ortega ${ }^{1}$, E. Collantes ${ }^{1}$, A. Escudero', R. Lopez-Mejías ${ }^{2}$, M.A. Gonzalez-Gay², C. Perez-Sanchez ${ }^{1}$. ${ }^{1}$ IMIBIC/Reina Sofía Hospital/University of Córdoba, Córdoba; ${ }^{2}$ Hospital Universitario Marqués de Valdecilla. IDIVAL. Santander. Universidad de Cantabria, Santander, Spain

Background: Circulating miRNAs have been proposed as attractive candidates as both diagnostic and prognostic biomarkers in various diseases, including a spectrum of autoimmune and cardiovascular conditions. Yet, the contribution of circulating miRNAs to the cardiovascular pathogenesis of Rheumatoid Arthitis (RA) patients and their potential role as biomarkers are still unknown.

Objectives: To identify circulating miRNAs as potential biomarkers of disease features and cardiovascular (CV) risk in RA

Methods: Plasma samples of 48 healthy donors (HDs) and 124 RA patients were collected. In the discovery phase, an array of 2083 human miRNAs was performed by using HTG EdgeSeq miRNA Whole Transcriptome Assay (Next generation sequencing) in 9 plasma samples (3 HDs, and 6 RA patients). Then, differentially expressed miRNAs, were selected and validated by RT-PCR in the whole cohort of patients and HDs. Potential targets of the validated miRNAs were identified by using Ingenuity Pathway Analysis (IPA) software and analysed at protein levels (Multiplex Assay). Correlation and association studies of altered miRNAs with analytical and clinical variables were also performed.

Results: The miRNA whole Transcriptome assay showed that 360 miRNAs were differentially expressed in RA patients in relation to HDs, including 261 upregulated and 97 downregulated. Functional classification (IPA) demonstrated that deregulated miRNAs were mainly involved in processes such as inflammatory response, connective tissue development and function, haematological disease, tissue development, and immunological disease. Nine microRNAs, selected among the most differentially expressed in the array, were selected for validation in all the subjects recruited (miR-299, miR-567, miR-4293, miR-135b, miR-6816, miR-346, miR-143, miR-199a, miR-106a, miR-148b). In silico analyses showed that these miRNAs had potential targets related to cytokine signalling, atherosclerosis pathway and intracellular signalling. The altered levels of selected miRNAs and its putative target proteins were validated in the whole cohort of patients.

A number of serum miRNAs in all the RA patients analysed were found interrelated and associated to autoimmunity (positivity for anti-CCPs and RF), bone erosion, inflammation (CRP, ESR, TNFa, IL6, IL8, IFN and IL-2), and evolution time. We could further identify a specific signature of four miRNAs (miR143, miR106, miR148b and miR567) that identified RA patients that had suffered previous CV events and specifically associated with the increase in the carotid intima-media thickness (CIMT) and with 'Framingham CV risk factors' such as diabetes, obesity or dyslipidemia.

Conclusions: We have branded novel and specific circulating miRNAs related to disease features and CV risk in RA patients, including, their autoimmune and inflammatory profile, the presence of Framingham risk factors and incipient atherosclerosis. These circulating miRNAs might be thus considered useful tools for the management of the disease in this autoimmune condition.

Acknowledgements: Funded by JA (CTS-7940) and the Ministry of Health (ISCIII, PI15/01333 and RIER RD16/0012/0015) cofinanced with FEDER funds.

Disclosure of Interest: None declared

DOI: 10.1136/annrheumdis-2018-eular.6814

\section{THU0067 ASSOCIATION OF HIGH TITERS OF ANTI- CARBAMYLATED PROTEINS ANTIBODIES WITH DECREASED BONE MINERAL DENSITY IN EARLY ARTHRITIS PATIENTS}

I. Arias de la Rosa ${ }^{1}$, A.M. Ortiz ${ }^{2}$, M.D. Boveda ${ }^{1}$, S. Castañeda ${ }^{2}$, I. GonzalezAlvaro', A. Gonzalez' ${ }^{1}{ }^{1}$ Instituto Investigacion Sanitaria- H. Clinico Universitario de Santiago, Santiago de Compostela; ${ }^{2}$ Hospital Universitario La Princesa - ISS-IP, Madrid, Spain

Background: Rheumatoid arthritis (RA) has a negative impact on bone homeostasis, in which multiple inflammatory mediators are involved together with the anti-citrullinated proteins antibodies (ACPA). These antibodies are able to promote osteoclastogenesis and bone loss even before the onset of synovitis Accordingly, ACPA are associated with the three aspects of bone loss: joint bone erosion, juxta-articular bone loss and, as recently demonstrated ${ }^{1}$, decreased bone mineral density (BMD). Other RA specific autoantibodies, the anti-carbamylated protein antibodies (anti-CarPA), are associated with the presence, severity and progression of erosions with independence of ACPA. However, their implication in the other aspects of bone loss has not been studied.

Objectives: To evaluate the association of anti-CarPA with BMD in a cohort of early arthritis (EA) patients.

Methods: Clinical data and samples were obtained at the first visit of 548 patients from the Princesa Early Arthritis Register Longitudinal (PEARL) study, which recruits patients within one year from arthritis onset. BMD was assessed by dualenergy x-ray absorptiometry (Hologic QDR-4500, Elite, Mass, USA) at the lumbar spine (LS), total hip (TH) and metacarpophalangeal (MCP) joints. BMD was considered as Z-scores, except for MCP due to lack of reference data. Anti-CarPA were determined by ELISA using carbamylated fetal calf serum. The ELISA results were considered as negative, below the 98 percentile of healthy controls, low-positive, below the median of positive patients, and high-positive, otherwise Data were analysed with linear regression including sex, age, BMI, and menopause as covariates.

Results: Anti-CarPA were positive in $25.9 \%$ of the EA patients. The positive patients did not show significant differences in BMD with the negative patients. 